\title{
Roughening of the anharmonic Larkin model
}

\author{
V. H. Purrello, ${ }^{1, *}$ J. L. Iguain,,${ }^{1 \dagger}$ and A. B. Kolton ${ }^{2, \dagger}$ \\ ${ }^{1}$ Instituto de Investigaciones Físicas de Mar del Plata (IFIMAR), Facultad de Ciencias Exactas y Naturales, Universidad Nacional de Mar \\ del Plata, Consejo Nacional de Investigaciones Científicas y Técnicas (CONICET), Deán Funes 3350, B7602AYL Mar del Plata, Argentina \\ ${ }^{2}$ Centro Atómico Bariloche and Instituto Balseiro, Comisión Nacional de Energía Atómica (CNEA), Consejo Nacional de Investigaciones \\ Científicas y Técnicas (CONICET), Universidad Nacional de Cuyo (UNCUYO), Av. E. Bustillo 9500, R8402AGP San Carlos de Bariloche, \\ Río Negro, Argentina
}

(Received 28 December 2018; published 5 March 2019)

\begin{abstract}
We study the roughening of $d$-dimensional directed elastic interfaces subject to quenched random forces. As in the Larkin model, random forces are considered constant in the displacement direction and uncorrelated in the perpendicular direction. The elastic energy density contains an harmonic part, proportional to $\left(\partial_{x} u\right)^{2}$, and an anharmonic part, proportional to $\left(\partial_{x} u\right)^{2 n}$, where $u$ is the displacement field and $n>1$ an integer. By heuristic scaling arguments, we obtain the global roughness exponent $\zeta$, the dynamic exponent $z$, and the harmonic to anharmonic crossover length scale, for arbitrary $d$ and $n$, yielding an upper critical dimension $d_{c}(n)=4 n$. We find a precise agreement with numerical calculations in $d=1$. For the $d=1$ case we observe, however, an anomalous "faceted" scaling, with the spectral roughness exponent $\zeta_{s}$ satisfying $\zeta_{s}>\zeta>1$ for any finite $n>1$, hence invalidating the usual single-exponent scaling for two-point correlation functions, and the small gradient approximation of the elastic energy density in the thermodynamic limit. We show that such $d=1$ case is directly related to a family of Brownian functionals parameterized by $n$, ranging from the random-acceleration model for $n=1$ to the Lévy arcsine-law problem for $n=\infty$. Our results may be experimentally relevant for describing the roughening of nonlinear elastic interfaces in a Matheron-de Marsilly type of random flow.
\end{abstract}

DOI: 10.1103/PhysRevE.99.032105

\section{INTRODUCTION}

The general study of elastic interfaces in random media is relevant for understanding generic properties displayed by a variety of experimental systems, and to successfully classify them into universality classes [1,2]. Disorder adds pinning, metastability, and generates macroscopically rough interfaces, while the elasticity tends to flatten them. When driven by a uniform force $F$, extended interfaces display a nontrivial zero-temperature depinning transition from a static to a sliding regime at a threshold value $F_{c}[3,4]$, while for finite temperatures a collective thermally activated creep motion persists below the depinning threshold, $F<F_{c}$ [4-7]. At zero force, the interface gets trapped in one of the many available deep metastable states $[8,9]$. In all cases, disorder induces statistically self-affine rough geometries in the putative steady states.

Universality allows convenient minimalistic models to capture the relevant disorder-elasticity interplay. A rather minimalistic, yet nontrivial model, is the one describing the position of a directed elastic interface at a time $t$ as an univalued scalar displacement field $u(x, t)$, where $x \in \mathbb{R}^{d}$, with $d$ the internal dimension of the manifold ( $d=0$ a particle, $d=1$ for a string, $d=2$ for a sheet, etc.), $D=d+1$ being the dimension of the space. Such elastic interfaces do not allow the formation of overhangs nor pinch-off bubbles. Specifically, we consider an elastic interface, subject to a pinning force $g[u(x, t), x]$

\footnotetext{
*vpurrello@ifimar-conicet.gob.ar

†iguain@mdp.edu.ar

koltona@cab.cnea.gov.ar
}

with a given elastic energy $E_{\mathrm{el}}(u)$. If we assume a nondriven overdamped relaxational dynamics at zero temperature, then the equation of motion of the interface results in

$$
\partial_{t} u(x, t)=-\frac{\delta E_{\mathrm{el}}}{\delta u(x, t)}+g[u(x, t), x] .
$$

A rather generic disorder is specified by the average over disorder realizations of the pinning force $\overline{g(x)}=0$, and its spatial autocorrelation function $\overline{g(x, u) g\left(x^{\prime}, u^{\prime}\right)}=\kappa^{2} \delta(x-$ $\left.x^{\prime}\right) \Delta_{\xi}\left(u-u^{\prime}\right)$. Here, $\Delta_{\xi}(u)$ is some even function of $u$ with $\xi$ denoting its shortest characteristic length. If we choose $\Delta_{\xi}(0)=1$, then $\kappa$ measures the strength of the disorder (see, for instance, Ref. [4]).

The simplest harmonic form $E_{\mathrm{el}}=\int d x\left(c_{2} / 2\right)\left(\partial_{x} u\right)^{2}$, with $c_{2} \geqslant 0$ the elastic constant, leads to the celebrated (zerotemperature) quenched Edwards-Wilkinson (QEW) equation [10]:

$$
\partial_{t} u(x, t)=c_{2} \partial_{x}^{2} u+g[u(x, t), x] .
$$

The main difficulty of Eq. (2) is the nonlinearity and heterogeneity of the pinning forces $g(u, x)$. This has led Larkin and coworkers $[8,11]$ to approach the problem perturbatively in the disorder such that, at first order, the pinning force can be simply approximated by an $x$-dependent but $u$-independent quenched random force, $g[u(x, t), x] \approx f(x)$, with

$$
\overline{f(x)}=0, \quad \overline{f(x) f\left(x^{\prime}\right)}=\kappa^{2} \delta\left(x-x^{\prime}\right) .
$$

Replacing $g$ by $f$ in Eq. (2), we obtain the linear equation

$$
\partial_{t} u(x, t)=c_{2} \partial_{x}^{2} u+f(x)
$$


governing the dynamics of the so-called Larkin model (LM). Since Eq. (4) is translationally invariant, bulk disorder does not pin the interface. However, it makes the interface evolve to a rough steady state.

In spite of its simplicity, the LM has many interesting properties and is a fundamental model in the theory of disordered elastic systems. The linear Eq. (4) is known to correctly describe the behavior of the QEW interfaces, which evolve according to the nonlinear Eq. (2), at scales smaller than the so-called Larkin length $L_{c} \sim(c \xi / \kappa)^{\frac{2}{4-d}}$, for $d<$ 4. Above $L_{c}$ the LM solution crossovers to the so-called random-manifold regime. Beyond the LM description, further crossovers at larger scales are still possible, depending on the properties of $\Delta_{\xi}(u)$, and also on the drive $F[4,7,8,12]$. In any case, the LM is relevant to estimate the fundamental physical units for expressing the large-scale solution of the full QEW problem. Quite remarkably, $L_{c}$ can be related to the macroscopic critical depinning threshold $F_{c} \sim \kappa / L_{c}^{d / 2}$, the elementary pinning energy barrier $U_{c}=F_{c} L_{c}^{d} \xi$, and the finite size crossover to metastability $[4,8,9]$. Above $L_{c}$, in the random-manifold regime, pinning and elastic energies scale in the same way as $\sim U_{c}\left(L / L_{c}\right)^{\theta}$, with $\theta$ a characteristic exponent, and the interface global width as $\xi\left(L / L_{c}\right)^{\zeta}$, with $\zeta$ a roughness exponent. It is worth also noting that the LM is relevant per se if the pinning forces have a large correlation length in the direction of interface displacements, such that $L_{c}$ is large compared to system size. This can be achieved if $\xi$ is very large, the system is elastically stiff or the disorder very weak. Such situations can arise experimentally, displaying a finite-size pinning crossover [13].

Interestingly, the physics of the LM is not only relevant for describing small length scales. Pinning forces such as $f(x)$ can be also generated via coarse-graining at large length scales $L \gg L_{c}$ and dominate over other forces in driven disordered elastic systems. This happens if the pinning force correlator $\Delta_{\xi}(u)$ has a periodicity [14]. One simple example is a QEW interface, Eq. (2), in a disordered medium with periodic boundary conditions in the direction of displacements, and an additional driving force $F$. Another less trivial example is a one-dimensional elastic chain of particles (or interfaces) with average separation $a_{0}$, driven in a completely uncorrelated random potential [15]. Such models are often used to study friction [16,17], but the physics is also relevant for charge density waves depinning $[14,18]$. In these cases, the largescale roughness is described by the solution of Eq. (4).

The LM being a fundamental model in the theory of disordered elastic systems, it is worth analyzing how their properties change under the influence of additional physically motivated terms in Eq. (4). In this paper, we focus in the influence of anharmonic corrections to the elasticity. To motivate the introduction of such corrections, we note that the LM, being linear, can be easily solved for a particular realization of $f(x)$ in any dimension $d$, and averages over realizations are straightforward. Much universal information can be obtained by studying the critical nonstationary relaxational dynamics, from a flat initial condition at the origin of displacements, i.e., $u(x, t=0)=0$. This process is dominated by a single dynamical growing length-scale $l(t)$, for long enough times before global equilibration.

We define the structure factor of the manifold as $S(q, t) \equiv \overline{|\hat{u}(q, t)|^{2}}$, where $\hat{u}(q, t)=L^{-d / 2} \int d x u(x, t) e^{-i q x}$ is the Fourier transform of $u(x, t)$. Using that $\hat{u}(q, t=0)=0$, we get

$$
S(q, t) \propto \frac{\kappa^{2}}{c_{2}^{2} q^{4}}\left(1-e^{-c_{2} q^{2} t}\right)^{2} .
$$

Then, the structure factor satisfies the general scaling $S(q, t) \sim q^{-(d+2 \zeta)} G\left(q t^{1 / z}\right)$, with $G(y)=y^{2\left(\zeta-\zeta_{s}\right)}$ for $y \gg 1$ and $G(y)=y^{d+2 \zeta}$ for $y \ll 1$ [19]. In our case, it is easy to check that the so-called global roughness exponent is $\zeta=$ $(4-d) / 2$ and coincides with the spectral roughness exponent $\zeta_{s}$, while the so-called dynamical exponent, related to the growing length-scale $l(t) \sim t^{1 / z}$, is $z=2$. In the steady state, roughly reached at times $t$ such that $l(t) \sim L, S(q, t \rightarrow$ $\infty) \sim q^{-(d+2 \zeta)}$. For an interface of size $L$, this gives the global squared width with respect to the center of mass position $W^{2} \equiv \overline{\left[u(x, t)-v_{\mathrm{cm}} t\right]^{2}}=\int d q S(q, t) \sim L^{2 \zeta}$, with $v_{\mathrm{cm}}=L^{-d} \int d x f(x) \sim L^{-d / 2}$ the finite-size residual center of mass velocity. The interface then becomes macroscopically self-affine, with exponent $\zeta$. That is, the rescaling $x^{\prime} \rightarrow b x$ and $u^{\prime} \rightarrow b^{\zeta} u$ leads to a statistically equivalent interface.

Interestingly, in the $d=1 \mathrm{LM}$, we have $\zeta_{s}=\zeta>1$. This situation, which has been called the super-rough case in Ref. [19], has a physical peculiarity. It implies that the harmonic elastic approximation in Eq. (4) to the local elastic couplings must break down in the thermodynamic limit, since $W / L \sim L^{\zeta-1} \rightarrow \infty$ as $L \rightarrow \infty$. Local elongations are thus not bounded in the thermodynamic limit. A similar situation occurs for the roughness exponent at the depinning threshold for the driven QEW model, where $\zeta \approx 1.25$ [20,21]. To remedy this situation, in Ref. [22] ad hoc anharmonic corrections to the elastic energy were introduced, such that

$$
E_{\mathrm{el}}(u)=\int d x\left[\frac{c_{2}}{2}\left(\partial_{x} u\right)^{2}+\frac{c_{2 n}}{2 n}\left(\partial_{x} u\right)^{2 n}\right],
$$

with $n=2,3,4 \ldots$, and constants $c_{2}>0, c_{2 n}>0$. It is worth noting that this kind of correction is Hamiltonian, convex, and being a correction to the elasticity only, translational invariant. In particular, note that the presence of the anharmonic correction breaks the tilt-symmetry of the full QEW equation, since both $g(u, x)$ and the harmonic elasticity, in contrast with the $\left(\partial_{x} u\right)^{2 n}$ term for $n>1$, are statistically invariant by the transformation $u \rightarrow u-s x_{\alpha}$, with $\alpha$ denoting any of the $d$ internal directions and $s$ the parameter measuring the tilt deformation.

For large $n$, Eq. (6) is equivalent to impose a hardconstraint to local elongations, an usual modeling of directed polymers [23]. The proposed nonquadratic term succeeds to save the elastic approximation in the thermodynamic limit at the depinning transition of the QEW model, with a new ("physical") roughness exponent $\zeta=\zeta_{s} \approx 0.63$ for all $n>1$ $[7,22]$. Quite remarkably, this value is in perfect agreement with the roughness at the depinning transition of the paradigmatic quenched Kardar-Parisi-Zhang (QKPZ) model [10], which is inherently a nonequilibrium effective equation that cannot be derived directly from a Hamiltonian or free energy. The anharmonic model hence allows us to study a model with a Hamiltonian, and a well-defined equilibrium state at $F=0$, which nevertheless spontaneously generates the ubiquitous Kardar-Parisi-Zhang (KPZ) [24] term, when it is driven by 
a force $F$. Thus, one may ask whether the breakdown of the small gradient approximation in the elastic energy of the $d=$ 1 Larkin model [Eq. (4)] is, similarly, protected by introducing a nonlinear elasticity of the form proposed in Eq. (6), and how $\zeta$ and $\zeta_{s}$ would change upon its addition. Solving the resulting "anharmonic Larkin model" would also allow us to find a possibly modified Larkin length $L_{c}$ and related quantities, which are fundamental to estimate both the critical force and the crossover length to the random manifold regime, for the anharmonic depinning model defined by Eqs. (1) and (6). The depinning transition of such model was analyzed in Ref. [22].

Motivated by the above phenomenology, in this work, we study the Larkin model with anharmonic elasticity, for a general dimension $d$ and $n \geqslant 2$. We obtain the global roughness exponent as a function of $n$ and $d$, and also describe the crossover from harmonic to anharmonic regimes of roughness, when two terms, one with $n=1$ and another with $n>1$ coexist. For the special $d=1$ case, where the small elastic deformation approximation is compromised, we show that, unlike the depinning model, the anharmonic correction is never able to reduce $\zeta$ below unity, even in the large $n$ almost hard-constraint limit. Moreover, we show that for all $n>1$ the $d=1$ interface displays anomalous scaling properties, with $\zeta_{s}>\zeta \geqslant 1$, the so-called "faceted regime" in Ref. [19]. Interestingly, we show also that this anomalous case is closely connected to an $n$-parameterized family of Brownian functionals which interpolate between the random-acceleration process for $n=1$, to the arcsine law Lévy stochastic process, for $n=\infty$. For $d>1$, however, we find $\zeta \leqslant 1$.

This article is organized as follows: in Sec. II we describe the anharmonic Larkin model, the observables of interest, and the methods. In Sec. III we derive, via heuristic arguments, the global roughness and dynamical exponents, and also the harmonic-anharmonic crossover length. In Sec. IV, we numerically test these predictions for $d=1$, solving both the relaxational dynamics of an interface as a function of time, and the statics, for two different boundary conditions. In Sec. $\mathrm{V}$, we discuss the relation for the anomalous $d=1$ case with a family of Brownian functionals. Finally, in Sec. VI we present our conclusions along with new open questions, and we suggest some possible applications for our results.

\section{MODEL AND OBSERVABLES}

We consider the anharmonic Larkin model using Eq. (1) in the Larkin approximation $g(u, x) \rightarrow f(x)$ and Eq. (6) for the elastic energy. The resulting equation of motion reads

$$
\partial_{t} u(x, t)=c_{2} \partial_{x}^{2} u+c_{2 n} \partial_{x}\left[\left(\partial_{x} u\right)^{2 n-1}\right]+f(x),
$$

where $n>1$. We will call Eq. (7) the "anharmonic Larkin model" (ALM). Fig. 1 schematically represents the model for the particular $d=2$ case.

Let us consider an interface of linear dimension $L$. The time-dependent global width for the relaxation process $W(L, t)$ is defined by the mean-squared fluctuations around the center of mass,

$$
W^{2}(L, t)=\overline{\frac{1}{L^{d}} \int d x\left(u-v_{\mathrm{cm}} t\right)^{2}},
$$

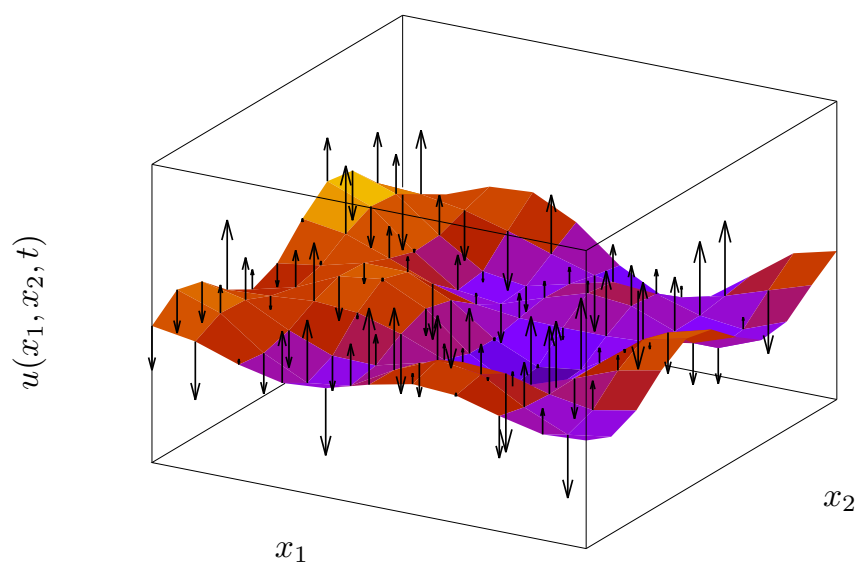

FIG. 1. Schematics of the anharmonic Larkin model (ALM) for the particular $d=2$ case, corresponding to a two-dimensional interface in a three-dimensional medium. The directed interface is described by an instantaneous scalar displacement field in the vertical direction $u(x, t)$ governed by Eq. (7), with $x \equiv\left(x_{1}, x_{2}\right)$ the internal coordinate. The elasticity of the interface is nonlinear, with an elastic energy given by Eq. (6). Arrows indicate that each point $x$ of the interface is subject to a quenched uncorrelated scalar random force $f(x)$, described by Eq. (3).

where $v_{\mathrm{cm}}=L^{-d} \int d x f(x) \sim L^{-d / 2}$ [from Eq. (7)] is the finite-size disorder-dependent but constant center of mass velocity, that may be observed for periodic or free boundary conditions. It is also useful to compute the time-dependent structure factor $S(q, t, L)$, as the interface displacement spatial power spectrum,

$$
S(q, t, L)=\overline{|\hat{u}(q, t)|^{2}},
$$

with $\hat{u}(q, t)$ the Fourier transform of $u(x, t)$ as defined just before Eq. (5).

Solving Eq. (7) from a flat initial condition $u(x, t=0)=0$ allows us to extract several universal exponents characterizing the generically expected critical relaxational dynamics. Such dynamics are expected to be controlled by a single growing correlation length scale,

$$
l(t) \sim t^{1 / z},
$$

with $z$ defining the dynamical exponent. Such scaling is expected to be valid at times larger than the microscopic time scale, but smaller than the time at which the system becomes completely correlated, i.e., when $l(t) \sim L$. However, from $W^{2}(L, t)$, and $S(q, t, L)$ we can define in principle three different roughness exponents $\left(z, \zeta\right.$, and $\left.\zeta_{s}\right)$ characterizing the random geometry below $l(t)$ [19].

The global roughness exponent $\zeta$ is defined from

$$
W(t, L) \sim t^{\zeta / z} \tilde{W}[L / l(t)]
$$

with

$$
\tilde{W}(x) \sim \begin{cases}x^{\zeta} & \text { if } x \ll 1 \\ \text { const } & \text { if } x \gg 1,\end{cases}
$$

or more directly from the stationary interface width

$$
W(t \rightarrow \infty, L) \sim L^{\zeta} .
$$


Both the global $\zeta$ and the spectral $\zeta_{s}$ roughness exponents can be obtained from the expected scaling

$$
S(q, t)=q^{-(2 \zeta+1)} \tilde{S}\left(q t^{1 / z}\right),
$$

where $\tilde{S}(x)$ is the scaling function and has the general form

$$
\tilde{S}(x) \sim \begin{cases}x^{2\left(\zeta-\zeta_{s}\right)}, & \text { if } x \gg 1 \\ x^{2 \zeta+1}, & \text { if } x \ll 1 .\end{cases}
$$

The stationary limit is reached when the correlation length $l(t)$ reaches $L$, at times of order $L^{z}$. Thus,

$$
S(q) \sim q^{-\left(2 \zeta_{s}+1\right)} L^{2\left(\zeta-\zeta_{s}\right)} .
$$

At zero temperature, the nonstationary scaling behavior from Eqs. (11), (13), (14), and (15), and also the stationary scaling described by Eq. (16), are verified by the QEW [Eq. (2)] at or above the depinning threshold $F \geqslant F_{c}[25,26]$, and in particular by the harmonic Larkin model [Eq. (4)] for any force, as shown in Eq. (5). In all these cases, a single growing correlation length controls the relaxation toward a unique self-affine stationary state, without memory of the initial condition. Different roughness exponents are obtained in each case. For instance, the $d=1$ QEW equation at $F_{c}$ has $\zeta=\zeta_{s} \approx 1.25$ and $z \approx 1.433$ [21], while at $F>F_{c}$, it crossovers to the Edwards-Wilkinson exponents $\zeta=\zeta_{s}=1 / 2$ and $z=2$. The harmonic Larkin model, however, as discussed in Sec. I, has $\zeta=\zeta_{s}=(4-d) / 2$ and $z=2$. In the following sections, we will show that the ALM also displays the same scaling forms, and we will obtain their exponents $\zeta, \zeta_{s}$, and $z$ for $n=1,2, \ldots, \infty$.

\section{SCALING ARGUMENTS}

The dynamical and roughness exponents of the EW, the LM and other linear models can be successfully obtained by simple scaling arguments (see Ref. [10] for many more relevant examples). Although such approach may fail in general (for instance, KPZ, QKPZ, or QEW equations require renormalization group calculations instead), we will employ the same naive methodology for the ALM nonlinear dynamics in Eq. (7). For simplicity, at first we consider only the nonlinear elastic term. Putting $c_{2}=0$, Eq. (7) reduces to

$$
\partial_{t} u(x, t)=c_{2 n} \partial_{x}\left[\left(\partial_{x} u\right)^{2 n-1}\right]+f(x) .
$$

Hence, to obtain the scaling exponents, we follow the standard naive procedure, which consists in rescaling space and time as

$$
\begin{aligned}
& x \rightarrow x^{\prime} \equiv b x, \\
& u \rightarrow u^{\prime} \equiv b^{\zeta} u, \\
& t \rightarrow t^{\prime} \equiv b^{z} t .
\end{aligned}
$$

Then, making the strong assumption that the elasticity and pinning parameters are not changed by rescaling, after this transformation, Eq. (17) results in

$$
b^{\zeta-z} \partial_{t} u=b^{(\zeta-1)(2 n-1)-1} c_{2 n} \partial_{x}\left[\left(\partial_{x} u\right)^{2 n-1}\right]+b^{-d / 2} f,
$$

where we have used that the random uncorrelated pinning forces scale as a $d$-dimensional random walk.
We finally require the resulting equation must be invariant under the transformation, which leads to the Flory-like exponents,

$$
\begin{gathered}
\zeta(d, n)=\frac{4 n-d}{4 n-2}, \\
z(d, n)=\zeta(d, n)+\frac{d}{2},
\end{gathered}
$$

for $n=1,2, \ldots, \infty$, and general dimension $d$.

As expected, for $n=1$, we recover the LM exponents, $\zeta=$ $(4-d) / 2$ and $z=2$, and the known upper critical dimension $d_{c}(n=1)=4$. From the prediction in Eqs. (22) and (23), it is worth noting the following:

(1) The upper critical dimension increases with $n$, as $d_{c}(n)=4 n$.

(2) In the hard-constraint case, corresponding to $n \rightarrow \infty$, we get, for a fixed dimension $d$, that $\zeta \rightarrow 1$, and $z \rightarrow 1+$ $d / 2$.

(3) The anharmonic elastic energy density scales as $L^{2 n[\zeta(d, n)-1]}$, thus for $n>1$ it is thermodynamically bounded only for $d>2$, since $\zeta(d>2, n)<1$. The $d=2$ case is always marginal, $\zeta(d=2, n)=1$, while the $d<2$ case diverges, since $\zeta(d<2, n)>1$.

(4) The spectral roughness exponent $\zeta_{s}$ does not come out from the arguments.

If the predicted exponents are valid, then we arrive to the striking conclusion that the unbounded local displacements predicted for the $d=1 \mathrm{LM}$ remain unbounded, in spite of the anharmonic elasticity, for any value of $n$. Therefore, the anharmonic correction to the elasticity can not save the small local deformation assumption behind the gradient expansion of the elastic energy density, even in the $n \rightarrow \infty$ hard-constraint limit, in the large-size limit. This is in sharp contrast to what happens for the $d=1$ QEW equation at depinning, where the elastic approximation is saved by the very same correction, making the depinning roughness exponent to change from $\zeta_{\text {dep }} \approx 1.25$ to the $n$-independent $(n>1)$ value $\zeta_{\text {dep }} \approx 0.63$ [22,27].

Let us now consider both the usual harmonic elasticity term and the nonlinear correction together, i.e., $c_{2}>0$ and $c_{2 n}>0$, for a given $n>1$, in Eq. (7). In this case, the harmonic term should dominate the behavior for small distortions, so there might be an anharmonic crossover length $L_{\text {anh }}$ between two regimes of roughness, from the harmonic $\mathrm{LM}\left(c_{2}>0, c_{2 n}=\right.$ $0)$ to the previously analyzed purely anharmonic $\operatorname{ALM}\left(c_{2}=\right.$ $0, c_{2 n}>0$ ) universality classes. Heuristically, we can propose that, for a length-scale $l>L_{\text {anh }}$, the purely anharmonic ALM displacement behaves as $u \sim u_{\text {anh }}\left(l / L_{\text {anh }}\right)^{\zeta(d, n)}$; while for $l<L_{\text {anh }}$, we have the harmonic result $u \sim\left(\kappa / c_{2}\right) l^{\zeta(d, n=1)}=$ $\left(\kappa / c_{2}\right) l^{(4-d) / 2}$. Sharply matching these two regimes at $L_{\text {anh }}$ implies

$$
u_{\mathrm{anh}}=\frac{\kappa}{c_{2}} L_{\mathrm{anh}}^{(4-d) / 2} .
$$

We propose that the crossover occurs when the two elastic energy terms are equally important, $c_{2}\left(u_{\mathrm{anh}} / L_{\mathrm{anh}}\right)^{2}=$ $\left(c_{2 n} / n\right)\left(u_{\mathrm{anh}} / L_{\mathrm{anh}}\right)^{2 n}$, thus,

$$
u_{\mathrm{anh}}=\left(\frac{n c_{2}}{c_{2 n}}\right)^{\frac{1}{2(n-1)}} L_{\mathrm{anh}}=\frac{\kappa}{c_{2}} L_{\mathrm{anh}}^{(4-d) / 2},
$$


which leads to the crossover length,

$$
L_{\text {anh }}=\left(\frac{c_{2}}{\kappa}\right)^{\frac{2}{2-d}}\left(\frac{n c_{2}}{c_{2 n}}\right)^{\frac{1}{(2-d)(n-1)}} .
$$

This means that, for the combined elasticities, we would have, in the stationary limit of a large system $L \gg L_{\text {anh }}$, a crossover behavior as a function of the length-scale in the correlation functions. For instance, the mean-square width [Eq. (8)] is expected to satisfy

$$
W^{2}(L, t \rightarrow \infty)=L^{2 \zeta(d, n=1)} w\left(L / L_{\mathrm{anh}}\right),
$$

with $w(x)=x^{2[\zeta(d, n>1)-\zeta(d, n=1)]}$, for $x \gg 1 ;$ and $w(x) \sim$ constant, for $x \ll 1$.

In the following two sections, we numerically check all these heuristic scaling predictions, for the special $d=1$ case.

\section{NUMERICAL RESULTS FOR $\boldsymbol{d}=1$}

To test the validity and robustness of the scaling predictions, we have found convenient to analyze separately the static solution, using appropriate boundary conditions. With these numerical methods, we are able to access all the exponents $\zeta, \zeta_{s}$, and $z$, and the crossover length $L_{\text {anh }}$.

\section{A. Static solution}

We study the static geometric properties of the ALM, by solving Eq. (7) in the stationary limit, where the elastic and random forces exactly balance, i.e.,

$$
c_{2} \partial_{x}^{2} u+c_{2 n} \partial_{x}\left[\left(\partial_{x} u\right)^{2 n-1}\right]=-f(x) .
$$

\section{Global roughness exponent}

Since the anharmonic term dominates the geometry at large length scales, we first focus on the purely anharmonic ALM by fixing $c_{2}=0$ in Eq. (28). This is then straightforward to solve:

$$
u(x)=-\int_{0}^{x} d x^{\prime}\left[\int_{0}^{x^{\prime}} d x^{\prime \prime} \frac{f\left(x^{\prime \prime}\right)}{c_{2 n}}-\left(\partial_{x} u(0)\right)^{2 n-1}\right]^{\frac{1}{2 n-1}},
$$

where we have used the particular boundary condition $u(0, t)=0$. As elastic interactions are short ranged, this particular boundary condition does not alter the large-scale scaling properties we are interested in.

To numerically evaluate Eq. (29), we simply discretize the $d=1$ interface in segments of size $\delta x$ and perform the sums

$$
u_{i}=-\sum_{j=0}^{i-1}\left[\sum_{k=0}^{j} \frac{f_{k}}{c_{2 n}}\right]^{\frac{1}{2 n-1}}, \quad \text { for } i>0,
$$

with $u_{0}=0$ fixed and $f_{0} \equiv-\sum_{i=1}^{L} f_{i}$; taking care of the statics of the whole polymer of size $L$ in the discrete description, and accounting for the term $\left[\partial_{x} u(0)\right]^{2 n-1} \sim\left(u_{1}-u_{0}\right)^{2 n-1}=$ $-f_{0} / c_{2 n}$ in the continuum Eq. (29) [28]. Note that, without loss of generality, we have taken $\delta x=1$ exploiting that the pinning force is completely uncorrelated in the internal coordinate. We have drawn $f_{k}$ from a uniform distribution with zero mean and variance $\kappa^{2}=1 / 12$.

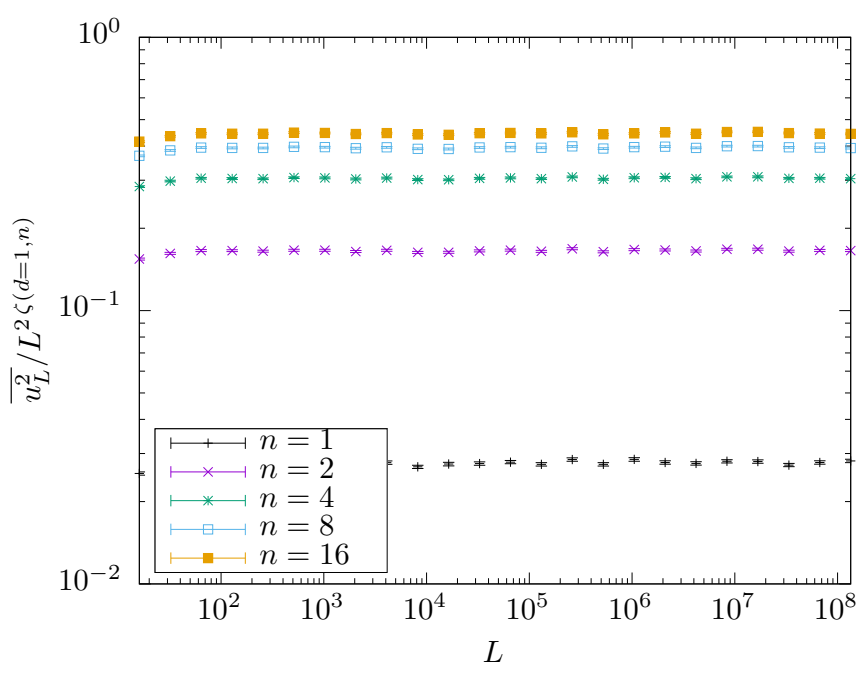

FIG. 2. Disorder-averaged last-end-point squared displacement of a $d=1$ ALM interface divided by the expected size scaling, as a function of the system size $L$, using $10^{4}$ samples for each value of $n$ and $L$. Constant behavior at large $L$ confirms the validity of the predicted global roughness exponent $\zeta(d=1, n)$ of Eq. (22). The error bars are estimated from the standard deviations.

Equation (30) can then be solved very efficiently for large system sizes, by using parallel random number generators and parallel prefix-sum algorithms implemented in massively parallel coprocessors, such as graphic cards. As the same scaling analysis leading to the prediction in Eq. (22) applies to Eq. (29) or Eq. (30), regardless of the fixed-end condition $u_{0}=0$, we expect to get $\zeta(d=1, n)$ from the numerical evaluation of Eq. (30). Since the system is not translational invariant along $x$, it is convenient to directly measure the last-point scaling, for which we expect $\overline{u_{L}^{2}} \sim L^{2 \zeta(d=1, n)}$, for large enough $L$.

In Fig. 2 we show there is an excellent agreement between predicted behavior and the obtained results for various values of $n$, for system sizes larger than around $10^{2}$, and up to $10^{8}$ string elements.

\section{Harmonic to anharmonic crossover}

To study the crossover between the LM and the ALM, we now consider the static condition Eq. (28), with $c_{2} \neq 0$ and $c_{2 n} \neq 0$, i.e., by including both the harmonic and the anharmonic terms. Integrating it, we get

$$
\begin{aligned}
c_{2} \partial_{x} u+c_{2 n}\left(\partial_{x} u\right)^{2 n-1}= & c_{2} \partial_{x} u(0)+c_{2 n}\left[\partial_{x} u(0)\right]^{2 n-1} \\
& -\int_{0}^{x} d x^{\prime \prime} f\left(x^{\prime \prime}\right)
\end{aligned}
$$

which is an odd $(2 n-1)$-degree polynomial in $\partial_{x} u$. Since $c_{2}>0$ and $c_{2 n}>0$, it has only one real root for each $x$. By integrating this $x$-dependent root we obtain $u(x)$. We perform this task numerically, for the discretized interface $u_{i}$, as before; using that $c_{2} \partial_{x} u(0)+c_{2 n}\left[\partial_{x} u(0)\right]^{2 n-1} \sim-f_{0}$ in this mixed case. Finally, to test the prediction for $L_{\mathrm{anh}}$ [Eq. (26)], we use the expected crossover scaling for the 


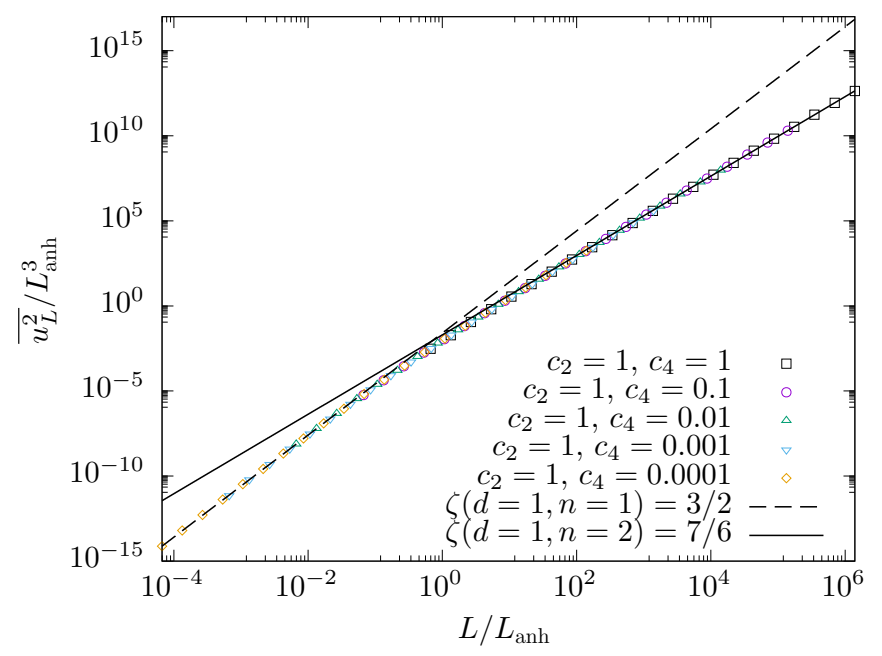

FIG. 3. Rescaled disorder-averaged quadratic displacement of the end-point combining both an harmonic and an anharmonic elasticity. The master curve confirms the general crossover scaling predicted in Eqs. (26) and (32), with $L_{\text {anh }}$ particularized for $d=$ 1 and $n=2$ [Eq. (33)]. The dashed and solid lines indicate the scalings $\overline{u_{L}^{2}} \sim L^{\zeta(d=1, n=1)}$ and $\overline{u_{L}^{2}} \sim L^{\zeta(d=1, n=2)}$, respectively, with the roughness exponents predicted by Eq. (22).

polymer endpoint,

$$
\overline{u_{L}^{2}} \sim L_{\mathrm{anh}}^{4-d} h\left(L / L_{\mathrm{anh}}\right),
$$

with $h(x) \sim x^{4-d}$, for $x \ll 1$; and $h(x) \sim x^{2 \zeta(d=1, n)}$, for $x \gg 1$.

We have tested the prediction for $L_{\text {anh }}$ from Eq. (26), for the particular $d=1$ and $n=2$ case, where Eq. (31) becomes an analytically solvable cubic polynomial. The prediction of Eq. (26) for this case is

$$
L_{\mathrm{anh}}=\left(\frac{c_{2}}{\kappa}\right)^{2} \frac{2 c_{2}}{c_{4}}=2 c_{2}^{2} \kappa^{-2} c_{4}^{-1} .
$$

In Fig. 3, we show the results for the rescaled families of curves, $\overline{u_{L}^{2}} / L_{\text {anh }}^{3}$ versus $L / L_{\mathrm{anh}}$, combining $c_{2}=1$ with various values for $c_{4}$, from $c_{4}=1$ to $c_{4}=0.0001$. The master curve strongly supports the validity of Eq. (26).

\section{B. Dynamic solution}

To test dynamical scaling (i.e., involving the time variable), we have performed numerical simulations of Eq. (7) in $d=1$ with periodic boundary conditions, and we have averaged the results over many disorder realizations. We have implemented this by using a spatial finite difference scheme, and we have solved the resulting system of equations following the standard Euler method. If the discretization is $\delta x$, such that $u_{i} \equiv u(x=i \delta x)$, for $i=1,2, \ldots, L$, then Eq. (7) can be approximated by

$$
\begin{aligned}
\partial_{t} u_{i}= & \frac{c_{2}}{(\delta x)^{3}}\left(u_{i+1}+u_{i-1}-2 u_{i}\right) \\
& +\frac{c_{2 n}}{(\delta x)^{2 n+1}}\left[\left(u_{i+1}-u_{i}\right)^{2 n-1}-\left(u_{i}-u_{i-1}\right)^{2 n-1}\right]+f_{i},
\end{aligned}
$$

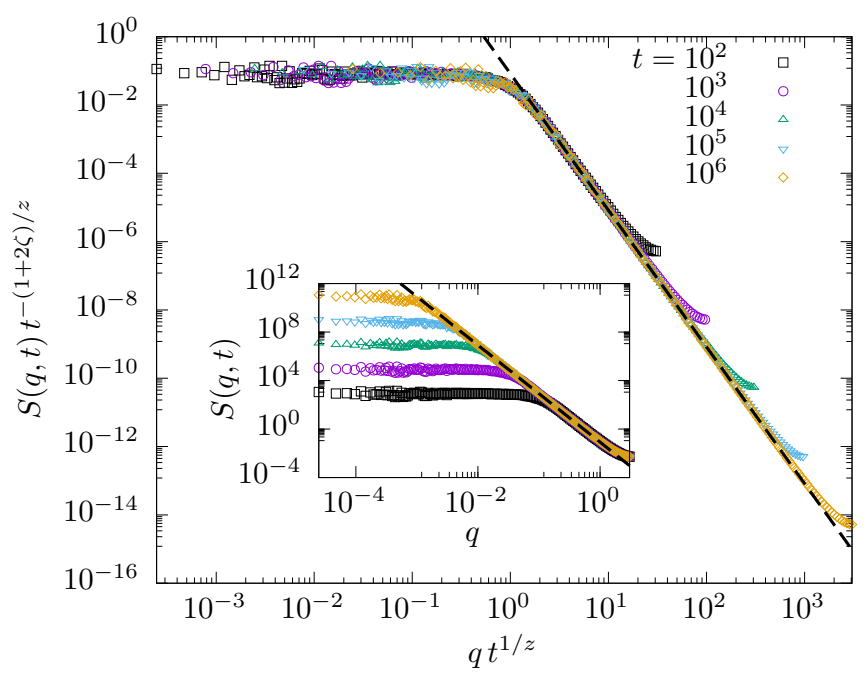

FIG. 4. Rescaled structure factors of the harmonic string (i.e., $d=1, n=1)$ Larkin model (LM) for different times $t$, rescaled according to Eq. (5) with $\zeta \equiv \zeta(d=1, n=1)=3 / 2$ and $z \equiv z(d=$ $1, n=1)=2$. The dashed line indicates a $q^{-\left(1+2 \zeta_{s}\right)}$ scaling, with $\zeta_{s}=\zeta$. Inset: raw data.

with $u_{0} \equiv u_{L}$ and $u_{L+1} \equiv u_{1}$. The $d$-dimensional generalization is straightforward. We draw the random forces $f_{i}$ from a uniform distribution with zero mean and variance $\kappa^{2}=$ $1 / 12$, and we solve Eq. (34) starting from a flat configuration $u_{i}(t=0)=0$.

\section{Time dependent structure factor}

The structure factor $S(q, t, L)$ allows us to obtain $\zeta$, and in particular, the dynamical exponent $z$ and the spectral roughness exponent $\zeta_{s}$, from the expected scaling of Eq. (14).

In Fig. 4 we show the corresponding $S(q, t)$ for $c_{2}=1$, $c_{2 n}=0, L=262144$, and different times $t$. The main panel shows the raw data (displayed in the inset) rescaled using Eq. (14). The result is perfectly consistent with the scaling and confirms the exponents $\zeta=3 / 2$ and $z=2$ of the analytical solution of Eqs. (22) and (23) for $d=1$ and $n=1$.

To contrast with the pure harmonic case considered before, we now consider the purely anharmonic case with $c_{2}=0$ and $c_{2 n}=1$ using $n=2$. In Fig. 5, we show the corresponding $S(q, t)$ for different times. Interestingly, we can observe that large $q$ behavior does not follow a master curve, but there is a downward drift by increasing times, which differs from the harmonic result shown in the inset of Fig. 4. Moreover, fitting the large $q$ power-law behavior for a long time $t$, yields a different power-law exponent than fitting the power-law envelope for all the times. In Fig. 6 we show that, nevertheless, the scaling of Eq. (14) works perfectly by using the global roughness exponent $\zeta(d=1, n=2)=7 / 6$ and the dynamical exponent $z(d=1, n=2)=5 / 3$, both predicted by dimensional analysis. However, in spite of the perfect collapse using the predictions of the scaling arguments of Eqs. (22) and (23) for $d=1$ and $n=2$, the master curve displays a clearly larger and well defined roughness exponent. From Eqs. (14) and (15), we can identify this new exponent with the spectral exponent $\zeta_{s}(d=1, n=2) \approx 1.39$. 


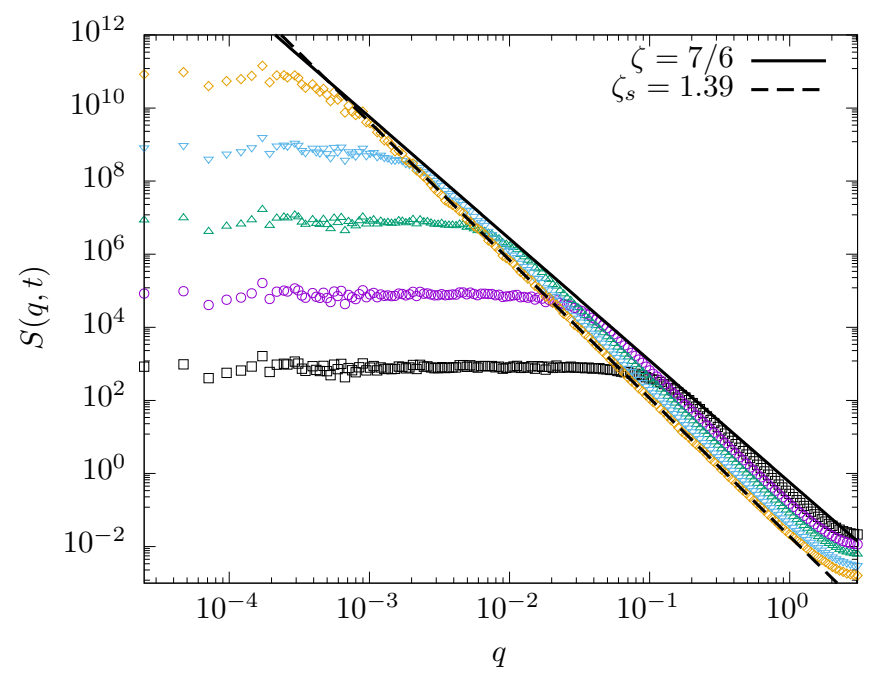

FIG. 5. Structure factor time evolution for the anharmonic string $n=2, d=1$. The spectral roughness exponent $\zeta_{s}$ fits individual curves as $S(q, t) \sim q^{-\left(1+2 \zeta_{s}\right)}$ for $q t^{1 / z} \gg 1$, while the global exponent $\zeta$ fits the curves envelope as $S(q, t) \sim q^{-(1+2 \zeta)}$. Scaling is anomalous, $\zeta_{s} \neq \zeta$, in contrast with the harmonic model (see Fig. 4).

\section{Critical exponents as a function of $\boldsymbol{n}$}

Repeating the above procedure, we have fitted $z, \zeta$, and $\zeta_{s}$ for different values of $n$, from $n=2$ to $n=100$. In Fig. 7 we show the corresponding results, where it can be appreciated that numerical simulations perfectly agree, within the error bars [29], with the predicted exponents $\zeta(d=1, n)$ and $z(d=$ $1, n$ ) (shown by solid lines), from Eqs. (22) and (23). In all cases, the fitted spectral exponent is found to be $\zeta_{s}(d=$ $1, n)>\zeta(d=1, n)$. We also observe that all exponents tend to converge to a well defined finite value in the large $n$ limit. The results also show a roughly constant difference, $\zeta_{s}(d=1, n)-\zeta(d=1, n) \approx 0.23$ for $n>1$, i.e., for all the

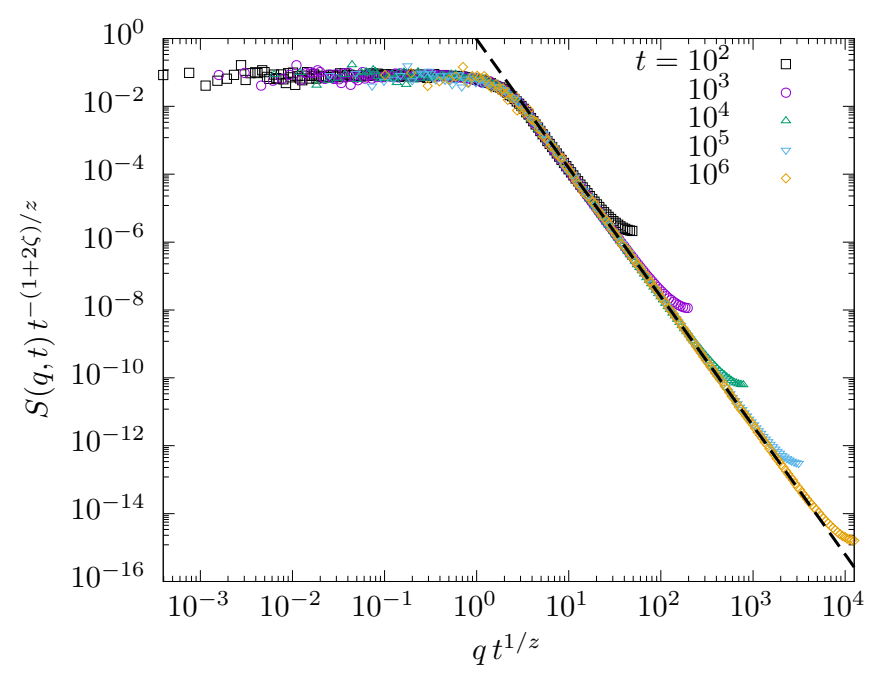

FIG. 6. Rescaled structure factor evolution for the $d=1$ and $n=2$ anharmonic Larkin model, using $\zeta \equiv \zeta(d=1, n=2)=7 / 6$ and $z \equiv z(d=1, n=2)=5 / 3$, as predicted by Eqs. (22) and (23). The dashed line corresponds to the power-law $1 / q^{1+2 \zeta_{s}}$, indicating a spectral roughness exponent $\zeta_{s} \approx 1.39$.

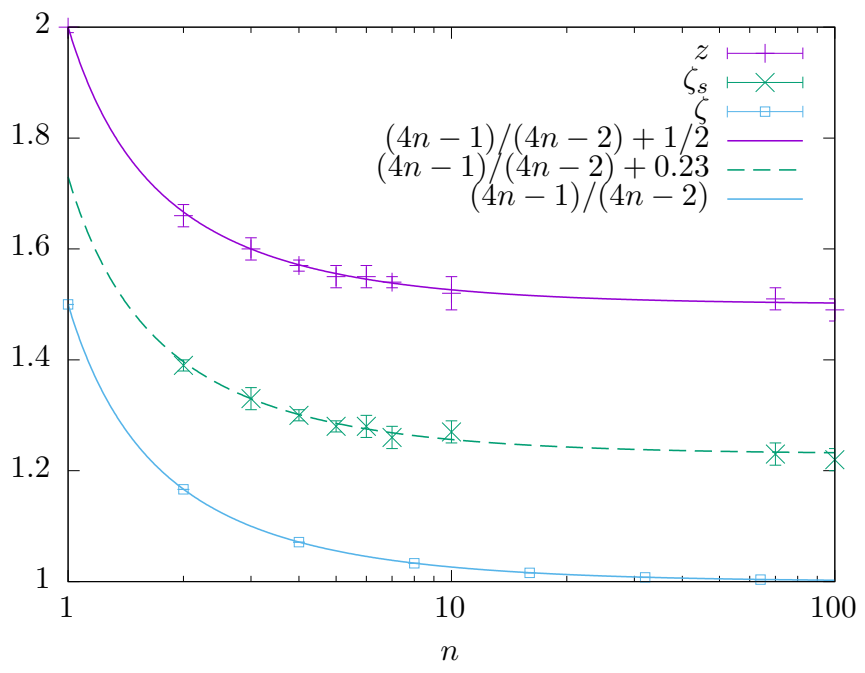

FIG. 7. Global roughness exponent $\zeta$, spectral roughness exponent $\zeta_{s}$, and dynamical exponent $z$ obtained from simulations of the $d=1$ harmonic and anharmonic Larkin models as a function of the elasticity exponent $n . n=1$ corresponds to the usual harmonic Larkin Model, while $n>1$ correspond to different anharmonic Larkin models. The spectral roughness exponent equals the global exponent only for the harmonic case, $n=1$. For $n>1$, we find $\zeta_{s}(d=1, n)-\zeta(d=1, n) \approx 0.23$, as indicated by the dashed line.

anharmonic cases. This is very interesting, as we do not have any analytical prediction for $\zeta_{s}(d=1, n)$.

\section{DISCUSSIONS}

We find an excellent agreement of the general scaling predictions of Sec. III with static and dynamical numerical simulations of a $d=1$ interface as a function of the anharmonicity parameter $n$. These predictions include the global roughness exponent $\zeta$, the dynamical exponent $z$, and the harmonic to anharmonic crossover length $L_{\text {anh }}$. We also obtain numerically the spectral roughness exponent $\zeta_{s}$, for which no analytical prediction is available, and we find that it is a different exponent, at least for $d=1$ and $n>1$. In the following sections we discuss possible connections of the ALM with other problems, and then we discuss the anomalous scaling.

\section{A. Family of Brownian functionals}

It is interesting to note that the static solution $u_{L}$ of Eq. (29) is directly related to a family of Brownian functionals [30] parametrized by $n$,

$$
u_{L}=-\int_{0}^{L} d x \operatorname{sign}[B(x)]|B(x)|^{\frac{1}{2 n-1}},
$$

with the Brownian path $B(x)$ given by

$$
B(x)=\int_{0}^{L} d x f(x) .
$$

From the statistical properties assumed for the elementary pinning forces [Eq. (3)], $B(x)$ is clearly a Wiener process. 


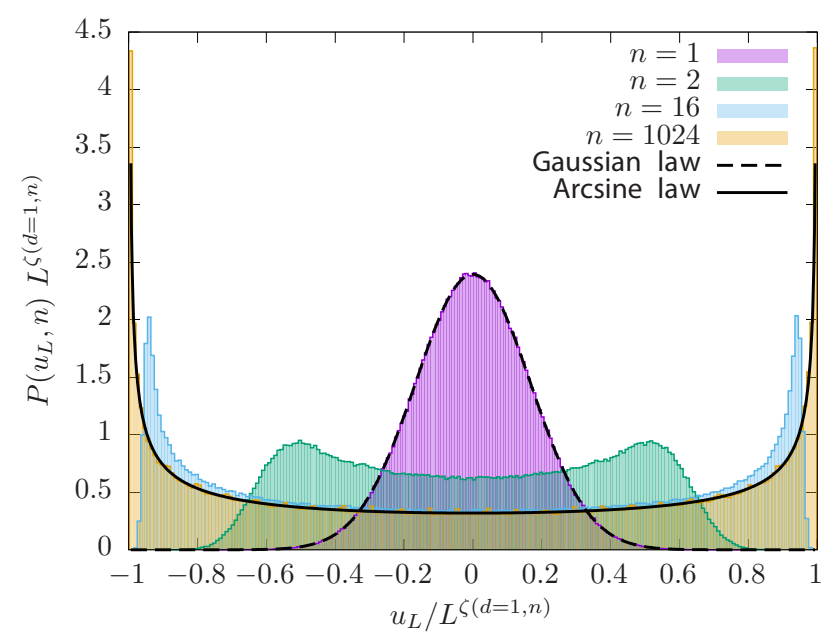

FIG. 8. Rescaled end-point distribution $P\left(u_{L}, n\right)$. The dashed and the solid lines indicate the analytical results known for the random acceleration process and for the Lévy arcsine-law process, respectively. The first maps to the harmonic Larkin model $(n=1)$; the second maps to the extreme $(n=\infty)$ anharmonic Larkin model.

Let us now analyze the family of Brownian functionals. The $n=1$ case,

$$
u_{L}=\int_{0}^{L} d x B(x)
$$

simply corresponds to the random-acceleration process (see, for instance, Refs. [31-33]). This well-known process has a Gaussian end-point distribution with a particular superextensive variance,

$$
P\left(u_{L}, n=1\right)=\frac{\exp \left[-u_{L}^{2} / 2 \sigma_{L}^{2}\right]}{\sqrt{2 \pi \sigma_{L}^{2}}}, \quad \sigma_{L}^{2} \sim L^{3} .
$$

Hence, $u_{L} \sim L^{3 / 2}$ is in perfect agreement with the scaling result $u_{L} \sim L^{\zeta}$, with the global roughness exponent $\zeta=\zeta(d=$ $1, n=1)=3 / 2$ predicted in Eq. (22).

On the other extreme of the ALM family, when $n \rightarrow \infty$ we get

$$
u_{L}=\int d x \operatorname{sign}[B(x)]
$$

which corresponds to the so-called arcsine-law Lévy process [34]. We have also an exact result for this case,

$$
P\left(u_{L}, n=\infty\right)=\frac{1}{\pi} \frac{1}{\sqrt{\left(L-u_{L}\right)\left(u_{L}+L\right)}} .
$$

Its variance grows as $\sim L^{2}$ implying that $u_{L} \sim L$, which is also in perfect agreement with our predicted $\zeta(d=1, n=\infty)=$ 1 in Eq. (22).

For intermediate cases, $1<n<\infty, P\left(u_{L}, n\right)$ is, to the best our knowledge, not known analytically. In Fig. 8 we show $P\left(u_{L}, n\right)$ obtained numerically from Eq. (30), for several values of $n$. These end-point distributions characterize the family of Brownian functionals describing the $d=1$ ALM. We observe that, in general, all $n>1$ distributions are symmetric but non-Gaussian, with twin peaks far from the origin that grow with increasing $n$, and diverge when $n \rightarrow \infty$, as the Lévy arcsine process is approached.

Different methods, such as the Feynman-Kac formula (see, for instance, Ref. [30]), could be used in principle to get $P\left(u_{L} ; n\right)$ analytically for general $n$, and to compare with our numerical results of Fig. 8 .

\section{B. Relation to variants of the KPZ equation}

Following the connection made in Ref. [22], between the QKPZ equation and the QEW equation with anharmonic elasticity at depinning, one may ask whether the ALM roughness properties can be related to other nonlinear roughening equations. At this respect, it is worth noting that the KPZ equation with temporally correlated noise,

$$
\partial_{t} u=c_{2} \partial_{x}^{2} u+\lambda\left(\partial_{x} u\right)^{2}+\eta(x, t),
$$

with the noise field such that $\langle\eta(x, t)\rangle=0$ and $\left\langle\eta(x, t) \eta\left(x^{\prime}, t^{\prime}\right)\right\rangle=\left|x-x^{\prime}\right|^{2 \psi-d}\left|t-t^{\prime}\right|^{2 \phi-1}$, was found to satisfy the scaling relation [10],

$$
z(1+2 \phi)-2 \zeta-d+2 \psi=0
$$

Interestingly, for quenched spatially uncorrelated noise [35] $(\phi=1 / 2$ and $\psi=0)$, we get $2 z-2 \zeta-d=0$, which is identical to Eq. (23). This can be related to the fact that Eq. (42) is derived from the nonrenormalization of the temporal component of the noise correlator, while Eq. (23) was derived assuming, analogously, the no-scaling of the random force amplitude. Since the global roughness and dynamic exponents of the ALM are parametrized by $n$, however, one can ask whether a value of $n$ exists such that the ALM and the quenched noise KPZ share the same exponents $\zeta$, $\zeta_{s}$, and $z$.

At this respect, numerical simulations of temporally correlated KPZ for $d=1$ and $\lambda>0$ report, for the $\phi=1 / 2$ quenched-noise limit, $\zeta \approx 1.06, z \approx 1.54$ [37] and $\zeta \approx 1.07$, $z \approx 1.15$ [36]. Exponents are thus roughly consistent with the $n \approx 4-5$ ALM, where $\zeta \approx 1.06 \pm 0.01$ and $z \approx 1.56$. Interestingly, in Ref. [36] it is shown that configurations display facets, with an spectral exponent $\zeta_{s} \approx 1.5$, which is slightly larger than the $\zeta_{s} \approx 1.29 \pm 0.01$ we find for $n \approx 4-5$ ALM. More recent results for the KPZ with temporally correlated noise approaching the limit $\phi=1 / 2$, report $\zeta_{s} \approx 1.3$, showing a closer agreement with the $n \approx 4-5$ universality class of the ALM [38]. The quenched noise KPZ with $\lambda<0$, on the other hand, has been associated (though for more general random forces) to faceted interfaces [39], similar to the ones we show for $d=1 \mathrm{in} \mathrm{Fig.} \mathrm{9.} \mathrm{The} \mathrm{possible} \mathrm{connection} \mathrm{of} \mathrm{the} \mathrm{ALM} \mathrm{for}$ general $n$ with $n$-dependent extensions of Eq. (41) remains an interesting open question.

Finally, it is also worth pointing out that the $d=1$ connection of the ALM with Brownian functionals of the previous section also suggests, from a different perspective, a connection with other KPZ variants. The Feynman-Kac formula is indeed connected to a family of quantum Hamiltonians describing a single particle in a family of potentials $p U(X)$ with $p$ a parameter (see, for instance, Ref. [30]). The quantum propagator satisfying each imaginary-time Schrödingerlike equation can then be mapped, by a suitable "ColeHopf" transformation, to a particular forced KPZ equation [40]. From Eq. (35) we have $U(X)=\operatorname{sign}[X]|X|^{\frac{1}{2 n-1}}$ so the 


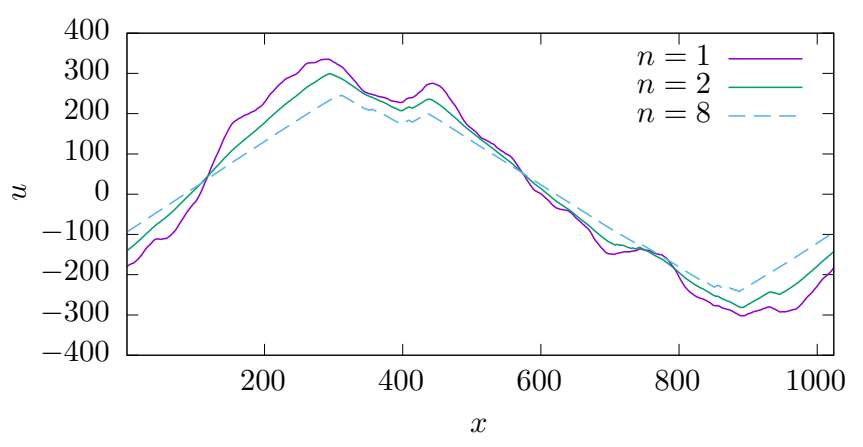

FIG. 9. Snapshots of $L=1024$ interfaces using the dynamic solution for different $n$ and the same realization of the random forces $f(x)$. The $n=1$ corresponds to the $1 d$ harmonic Larkin model. The interfaces become more "faceted" with further increasing $n$.

potential is parametrized by both $p$ and $n$. It would be interesting to test these connections further.

\section{Two different roughness exponents}

The ALM displays interesting anomalous scaling properties. In particular, in the steady state, the spectral roughness exponent does not coincide with the global roughness exponent $\left(\zeta_{s}>\zeta\right.$ for $\left.n>1\right)$ in $d=1$. This is in sharp contrast to what happens for the LM and many other linear surface growth models, such as the EW equation, where interfaces are characterized by a single roughness exponent, and the usual Family-Vicsek scaling applies [10]. It is worth noting, however, that also nonlinear models such as the KPZ equation with additive noise [41], or the zero temperature QEW equation at the depinning threshold [21], seem to display a normal Family-Vicsek scaling of the two point correlations functions, such that $\zeta \approx \zeta_{s}$, with $\zeta_{s} \approx 1 / 2$ in the former and $\zeta_{s} \approx 1.25$ in the latter, in $d=1$. Therefore, nonlinearity, either in the elasticity or in the disorder, is not sufficient to produce the anomaly, even combining it with super-rough behavior $\zeta_{s}>1$, as it occurs in the QEW at depinning. We also note that breaking the tilt symmetry (as for instance in the KPZ equation), or breaking the statistical tilt symmetry (as for instance in the $d=1 \mathrm{QKPZ}$ equation at depinning where $\zeta_{s} \approx$ $\zeta \approx 0.63[7,22])$, does not seem to be sufficient for producing the anomaly either.

However, other models are known to display the $\zeta \neq \zeta_{s}$ anomalous behavior. The anomalous case with $\zeta_{s}<1$, so called "intrinsic," has been, for instance, observed [42]. The case $\zeta_{s}>1$ has been observed, however, in the Sneppen model A of self organized criticality [19]. Interestingly, in Ref. [19], the $\zeta \neq \zeta_{s}>1$ behavior has been associated to generic "faceted" stationary interfaces, and a simple model of random \pm 1 slopes used to illustrate the association. In Fig. 9, we show that this is indeed qualitatively the case for the ALM family parameterized by $n$ in $d=1$. As far as we can tell, the generic origin of the $\zeta \neq \zeta_{s}$ behavior, either in the $\zeta_{s}<1$ ("intrinsic") or $\zeta_{s}>1$ ("faceted") remains open, however.

We speculate that the observed anomalous roughness property $\zeta \neq \zeta_{s}$ of the $d=1$ ALM should be related to the shape of $P\left(u_{L}, n\right)$ for $n>1$. Indeed, only for the LM $(n=1)$ the distribution is Gaussian and $\zeta=\zeta_{s}$. For $n>1$ it is non-
Gaussian and presents two twin peaks and, as expected, the larger and sharper they are, the more faceted the individual configurations look like. Since the non-Gaussian bimodal shape of $P\left(u_{L}, n\right)$ presents scale invariance, the associated faceted structure should be also scale invariant and thus visible in the structure factor shape. This makes us conjecture that $\zeta_{s}(d=1, n)$ must be somehow coded in $P\left(u_{L}, n\right)$. It would be useful to test this hypothesis for $d=1$ by analytical calculations using, for instance, Brownian functionals techniques.

For $d>1$, however, it would be useful to go beyond heuristic methods and use the Gaussian variational method (see, for instance, Ref. [43]), or renormalization group techniques, to calculate both $\zeta$ and particularly $\zeta_{s}$, which we were unable to estimate by simple scaling arguments. One important question is when $\zeta_{s} \neq \zeta$, and to know if $\zeta_{s}$ is universal. In other words, the question is whether $\zeta_{s}$ is needed to make a finer classification into (anomalous) universality classes.

\section{CONCLUSIONS}

We have studied the roughness and dynamical properties of the anharmonic Larkin model. By Flory-like scaling arguments, we have obtained the global $\zeta$ roughness exponent, the dynamic exponent $z$, and the harmonic to anharmonic crossover length scale for arbitrary both $d$ and $n$. An excellent agreement is obtained by comparing with numerical calculations in $d=1$, and we have showed that, in this case, the model directly relates to a family of Brownian functionals parameterized by $n$; ranging from the random-acceleration model $(n=1)$ to the Lévy arcsine-law problem $(n=\infty)$. It would be interesting to exploit this connection, not only to find analytical methods of solution but to link or map the $d=1$ ALM model to other problems.

From the analytical and numerical calculations, we have found an intriguing anomalous scaling for the $d=1 \mathrm{case}$. The spectral roughness exponent $\zeta_{s}$ is different from the global $\zeta$, and $\zeta_{s}>\zeta>1$, for $n>1$. On the one hand, this implies that the small gradient expansion for the elastic energy is always compromised in the thermodynamic limit, even in the $n \gg 1$ hard-constraint limit. On the other hand, that we need two roughness exponents, already at the level of the two point correlation functions, to describe the self-affine geometry of the interface. It would be interesting to see if the "faceted" scaling anomaly $\zeta_{s} \neq \zeta$ persists at larger dimensions, and in particular, to find an analytical estimate of $\zeta_{s}$ as a function of dimension $d$ and the anharmonicity parameter $n$ using, for instance, variational or renormalization group approaches. This would allows us to test the universality of $\zeta_{s}$, and to point out the mechanism originating the anomaly in general cases.

Finally, although the Larkin model is usually a local approximation for more realistic models of disordered elastic systems with many metastable states, it would be nevertheless interesting to check some of our results experimentally. This is in principle possible in systems with anisotropically correlated random forces. Indeed, interesting anomalous scaling was found for interfaces with "columnar noise," both theoretically [36] and experimentally [44]. Finite systems with either stiff elastic couplings or very weak disorder can also comply with the Larkin approximation for disorder at the relevant scales. Larkin random forces could be also spontaneously 
generated, by coarse-graining, well beyond the Larkin length in large driven periodic systems such as elastic chains [15]. In all these cases, the additional anharmonicity needed for realizing the ALM described here may arise from some nonlinear local interaction breaking the tilt symmetry of the clean (i.e., nondisordered) system. Directed polymers or membranes in a layered Matteron-de Marsily scalar flow field [45] may be a realization of the ALM schematically represented in Fig. 1.

\section{ACKNOWLEDGMENTS}

We thank S. Bustingorry, A. Rosso, C. Texier, and T. Giamarchi for useful and motivating discussions. This work was partly supported by Grants No. PICT20160069/FONCyT and No. UNCuyo C017 from Argentina. We have used Mendieta Cluster from CCAD-UNC and GPGPUCAB Cluster from CAB, which are part of SNCAD-MinCyT, Argentina.

\section{APPENDIX: LARKIN LENGTH WITH PURE NONHARMONIC ELASTICITY}

If we see Eq. (7) as a short-scale approximation of the full model of Eq. (1) with Eq. (6), then it is useful to get the corresponding ("anharmonic") Larkin length.
By balancing the elastic force with the pinning force of a piece of linear size $l$, we get $c_{2 n} u^{2 n-1} l^{-2(2 n-1)-1} \approx \kappa l^{-d / 2}$. We can define $L_{c}(d, n)$ as the length $l$ corresponding to $u=\xi$,

$$
\xi=\left(\frac{\kappa}{c_{2 n}}\right)^{\frac{1}{2 n-1}} L_{c}^{\zeta(d, n)}
$$

so we obtain

$$
L_{c}(d, n)=\left[\xi^{2 n-1}\left(\frac{c_{2 n}}{\kappa}\right)\right]^{2 /(4 n-d)} .
$$

Thus, for $n=1$, we recover the well-known harmonic result $L_{c}(d, 1)=\left(\xi c_{2} / \kappa\right)^{2 /(4-d)}$. For $n=\infty$, however, $L_{c} \rightarrow$ $\xi$. The length $L_{c}(d, n)$ marks the crossover to the randommanifold regime of the purely anharmonic interface. While the Larkin regime is described by the exponents $\zeta(d, n)$ [Eq. (22)] and $z(d, n)$ [Eq. (23)], regardless of the presence of the driving force $F$, the random-manifold exponents do change with $F$. The equilibrium random manifold exponents (present at equilibrium or in the creep regime at intermediate scales $[7,25]$ ) are expected to be the same as those for the harmonic elasticity or QEW equation, while the depinning random manifold exponents (present at depinning or in the creep regime at large scales for vanishing velocities [25,26]) coincide with the ones of the QKPZ equation [22].
[1] D. S. Fisher, Phys. Rep. 301, 113 (1998).

[2] M. Kardar, Phys. Rep. 301, 85 (1998).

[3] H. Leschhorn, T. Nattermann, S. Stepanow, and L. Tang, Ann. Phys. 509, 1 (1997).

[4] P. Chauve, T. Giamarchi, and P. Le Doussal, Phys. Rev. B 62, 6241 (2000).

[5] L. B. Ioffe and V. M. Vinokur, J. Phys. C 20, 6149 (1987).

[6] T. Nattermann, Y. Shapir, and I. Vilfan, Phys. Rev. B 42, 8577 (1990).

[7] A. B. Kolton, A. Rosso, T. Giamarchi, and W. Krauth, Phys. Rev. B 79, 184207 (2009).

[8] G. Blatter, M. V. Feigel'man, V. B. Geshkenbein, A. I. Larkin, and V. M. Vinokur, Rev. Mod. Phys. 66, 1125 (1994).

[9] T. Nattermann and S. Scheidl, Adv. Phys. 49, 607 (2000).

[10] A. Barabási and H. Stanley, Fractal Concepts in Surface Growth (Cambridge University Press, Cambridge, 1995).

[11] A. I. Larkin and Y. N. Ovchinnikov, J. Low Temp. Phys. 34, 409 (1979).

[12] T. Giamarchi and S. Bhattacharya, in High Magnetic Fields, edited by C. Berthier, L. P. Lévy, and G. Martinez, Lecture Notes in Physics Vol. 595 (Springer-Verlag, Berlin, 2002), pp. 314-360.

[13] M. I. Dolz, A. B. Kolton, and H. Pastoriza, Phys. Rev. B 81, 092502 (2010).

[14] P. Le Doussal, K. J. Wiese, and P. Chauve, Phys. Rev. B 66, 174201 (2002).

[15] S. Bustingorry, A. B. Kolton, and T. Giamarchi, Phys. Rev. B 82, 094202 (2010).

[16] B. N. J. Persson, Sliding Friction (Springer, Berlin, 2000).

[17] D. Cule and T. Hwa, Phys. Rev. B 57, 8235 (1998).

[18] S. Brazovskii and T. Nattermann, Adv. Phys. 53, 177 (2004).
[19] J. J. Ramasco, J. M. López, and M. A. Rodríguez, Phys. Rev. Lett. 84, 2199 (2000).

[20] A. Rosso, A. K. Hartmann, and W. Krauth, Phys. Rev. E 67, 021602 (2003).

[21] E. E. Ferrero, S. Bustingorry, and A. B. Kolton, Phys. Rev. E 87, 032122 (2013).

[22] A. Rosso and W. Krauth, Phys. Rev. Lett. 87, 187002 (2001).

[23] T. Halpin-Healy and Y.-C. Zhang, Phys. Rep. 254, 215 (1995).

[24] M. Kardar, G. Parisi, and Y.-C. Zhang, Phys. Rev. Lett. 56, 889 (1986).

[25] A. B. Kolton, A. Rosso, E. V. Albano, and T. Giamarchi, Phys. Rev. B 74, 140201 (2006).

[26] A. B. Kolton, G. Schehr, and P. Le Doussal, Phys. Rev. Lett. 103, 160602 (2009).

[27] Note that if the ALM is considered as a short-scale approximation of the QEW with anharmonic corrections [22], it applies only below the corresponding finite Larkin length, calculated in the Appendix. In this case, the elastic approximation is not necessarily compromised in the large scale limit in spite of the super-rough (anharmonic) Larkin regime.

[28] Note that the constraint force $f_{0}$, needed to fix the boundary conditions in the first displacement $u_{0}$ of the polymer, is not described by the bulk random forces, with the statistical properties in Eq. (3).

[29] We use different criteria to estimate the error bars for each exponent. In the case of $\zeta$, we estimate the uncertainty fitting the data of Fig. 2. The error bar corresponding to $\zeta_{s}$ is obtained by fitting the slope in the rescaled structure factors, shown in Fig. 6. For $z$ we make a conservative estimation of its uncertainty by determining the range where a good collapse can be seen. 
[30] S. N. Majumdar, Brownian functionals in physics and computer science, in The Legacy of Albert Einstein (World Scientific, Singapore, 2006), pp. 93-129.

[31] T. W. Burkhardt, J. Stat. Mech. (2007) P07004.

[32] H. J. Hilhorst, P. Calka, and G. Schehr, J. Stat. Mech. (2008) P10010.

[33] S. N. Majumdar, A. Rosso, and A. Zoia, J. Phys. A: Math. Theor. 43, 115001 (2010).

[34] P. Lévy, Compositio Mathematica 7, 283 (1940).

[35] M. E. Cates and R. C. Ball, J. Phys. France 49, 2009 (1988).

[36] I. G. Szendro, J. M. López, and M. A. Rodríguez, Phys. Rev. E 76, 011603 (2007).

[37] T. Song and H. Xia, J. Stat. Mech. (2016) 113206.
[38] A. Alés and J. M. López, arXiv:1902.06674.

[39] H. Jeong, B. Kahng, and D. Kim, Phys. Rev. E 59, 1570 (1999).

[40] M. Kardar, Statistical Physics of Fields (Cambridge University Press, Cambridge, 2007).

[41] S. Bustingorry, J. Stat. Mech. (2007) P10002.

[42] J. M. López, M. A. Rodríguez, and R. Cuerno, Phys. Rev. E 56, 3993 (1997)

[43] E. Agoritsas, V. Lecomte, and T. Giamarchi, Physica B: Condens. Matter 407, 1725 (2012).

[44] J. Soriano, J. J. Ramasco, M. A. Rodríguez, A. HernándezMachado, and J. Ortín, Phys. Rev. Lett. 89, 026102 (2002).

[45] G. Oshanin and A. Blumen, Phys. Rev. E 49, 4185 (1994). 